\title{
Visceral fat changes after distal gastrectomy according to type of reconstruction procedure for gastric cancer
}

Koji Tanaka*, Isao Miyashiro, Masahiko Yano, Kentaro Kishi, Masaaki Motoori, Tatsushi Shingai, Shingo Noura, Masayuki Ohue, Hiroaki Ohigashi and Osamu Ishikawa

\begin{abstract}
Background: Noncancerous causes of death, such as cerebrovascular or cardiac disease, are not rare in patients with gastric cancer who had undergone curative gastrectomy. Metabolic syndrome, characterized by visceral fat accumulation, is a risk factor for cerebrovascular and cardiac diseases. However, there is limited information on the effects of reconstruction procedures on changes in visceral fat after distal gastrectomy. The aim of this study was to analyze the impact of the reconstruction procedure (Roux-en-Y reconstruction (RY) and Billroth I reconstruction (BI)) on changes in visceral fat, as determined using computed tomography.
\end{abstract}

Methods: The study subjects were 152 patients with gastric cancer who underwent distal gastrectomy with lymphadenectomy between 2002 and 2007. The visceral fat area was measured for one cross-sectional computed tomogram obtained at the level of the umbilicus.

Results: Adjuvant chemotherapy (yes vs. no, $P=0.0006$ ), type of reconstruction ( $B \mid$ vs. $R Y, P=0.0146$ ), field of lymph node dissection ( $\angle \mathrm{D} 2$ vs. $\geq \mathrm{D} 2, P=0.0020$ ), omentectomy (yes vs. no, $P=0.0003$ ), and pathological stage (1/2 vs. 3/4; $P=0.0023)$ correlated significantly with postoperative visceral fat loss. Multivariate logistic regression analysis identified reconstruction ( $B I$ vs. RY; $P=0.0232$ ) and adjuvant chemotherapy (yes vs. no, $P=0.0330$ ) as the significant determinants of visceral fat loss after surgery.

Conclusions: Visceral fat loss after RY was larger than that after BI. Further prospective studies are needed to confirm the effects of reconstruction after distal gastrectomy on visceral fat.

Keywords: Gastrectomy, Reconstruction, Visceral fat

\section{Background}

Noncancerous death is a common cause of death in patients with gastric cancer who had undergone curative gastrectomy. This is probably because the 5-year survival rate has improved to about $95 \%$, especially in patients with early gastric cancer treated by radical resection [1]. The causes of noncancerous deaths include cerebrovascular, cardiac, and respiratory diseases [2]. The metabolic syndrome, characterized by visceral fat accumulation, is a risk factor for cerebrovascular and cardiac diseases [3-5]. The Roux-en-Y gastric bypass is a popular procedure used to reduce body weight, and visceral fat, in morbidly obese

\footnotetext{
* Correspondence: ktanaka@gesurg.med.osaka-u.ac.jp

Department of Surgery, Osaka Medical Center for Cancer and Cardiovascular Diseases, 1-3-3 Nakamichi, Higashinari-ku, Osaka 537-8511, Japan
}

subjects. Although Roux-en-Y (RY) gastric bypass and Roux-en-Y reconstruction after gastrectomy for gastric cancer are not entirely similar, the selection of reconstruction method could control visceral fat after gastrectomy for gastric cancer.

Several studies have reported poor prognosis of patients with excess post-gastrectomy body weight loss $[6,7]$. Body weight loss is a serious problem, especially for lean patients. Such weight loss is caused by impaired food intake and malabsorption [8-10] Body weight loss after gastrectomy is thought to be mainly due to loss of body fat (which includes subcutaneous and visceral fat) [11-13]. In this regard, visceral fat change is worthy of remark. However, to our knowledge, there is no information on changes in visceral fat and subcutaneous fat
C Bïomed Central

(C) 2013 Tanaka et al.; licensee BioMed Central Ltd. This is an Open Access article distributed under the terms of the Creative Commons Attribution License (http://creativecommons.org/licenses/by/2.0), which permits unrestricted use, distribution, and reproduction in any medium, provided the original work is properly cited. 
distribution after gastrectomy for different surgical procedures, such as distal gastrectomy with Billroth I (DGBI) reconstruction or distal gastrectomy with Roux-en-Y (DGRY) reconstruction.

In this retrospective study, we first quantified the visceral fat area (VFA) and then determined the impact of different surgical procedures (DGBI and DGRY) on the visceral fat of patients who underwent distal gastrectomy with lymphadenectomy. Clinically, this study serves to provide a basis for appropriate selection of reconstruction method following distal gastrectomy, to provide adequate control of visceral fat volume postoperatively in the long-term.

\section{Methods}

\section{Patients and surgical procedures}

Between January 2002 and April 2007, a total of 152 patients with gastric cancer underwent distal gastrectomy with lymphadenectomy at the Osaka Medical Center for Cancer and Cardiovascular Diseases, Osaka, Japan. They included 106 men and 46 women with a mean age of $62.8 \pm 9.8$ years $( \pm$ SD, range: 37 to 85 years; median: 63 years). All patients underwent preoperative assessment, including gastric endoscopy, computed tomography (CT) and laboratory tests. In this study, we compared the CT images taken at least 6 months after surgery with those before surgery.

Roux-en-Y reconstruction was performed when the remnant stomach after resection was too small to allow performance of $\mathrm{BI}$ or when the main tumor invaded the pylorus. Lymph node dissection was performed according to the second edition of the Japanese Classification of Gastric Cancer [14]. Specifically, patients with T1 and N0 tumors underwent dissection of the perigastric lymph nodes and nodes at the base of the left gastric artery, and along the common hepatic artery, which for simplicity is termed 'D1+ lymph node dissection' in this study. Patients with $\mathrm{T} 2$ or more advanced tumors and those with N1 or more advanced cancer underwent D2 lymph node dissection, which involves dissection of hepatoduodenal nodes and retropancreatic nodes.

Hospital records were reviewed for age, sex, height, body weight, clinicopathological background data regarding UICC-TNM stage, and surgical procedure. This study was approved by the Human Ethics Review Committee of the Osaka Medical Center for Cancer and Cardiovascular Diseases.

\section{Quantification of visceral fat area}

The VFA was measured using 'FatScan', as described previously [15], on one cross-sectional CT image obtained at the level of the umbilicus. Figure 1 shows the method used to determine the fat tissue area on a CT image. First, the intraperitoneal area was defined manually by tracing its contour on the scan (Figure 1a). Thereafter, a region of interest of the subcutaneous fat layer was defined by tracing its contour on each scan, either automatically or manually (Figure $1 \mathrm{~b}$ ), and then the attenuation range of
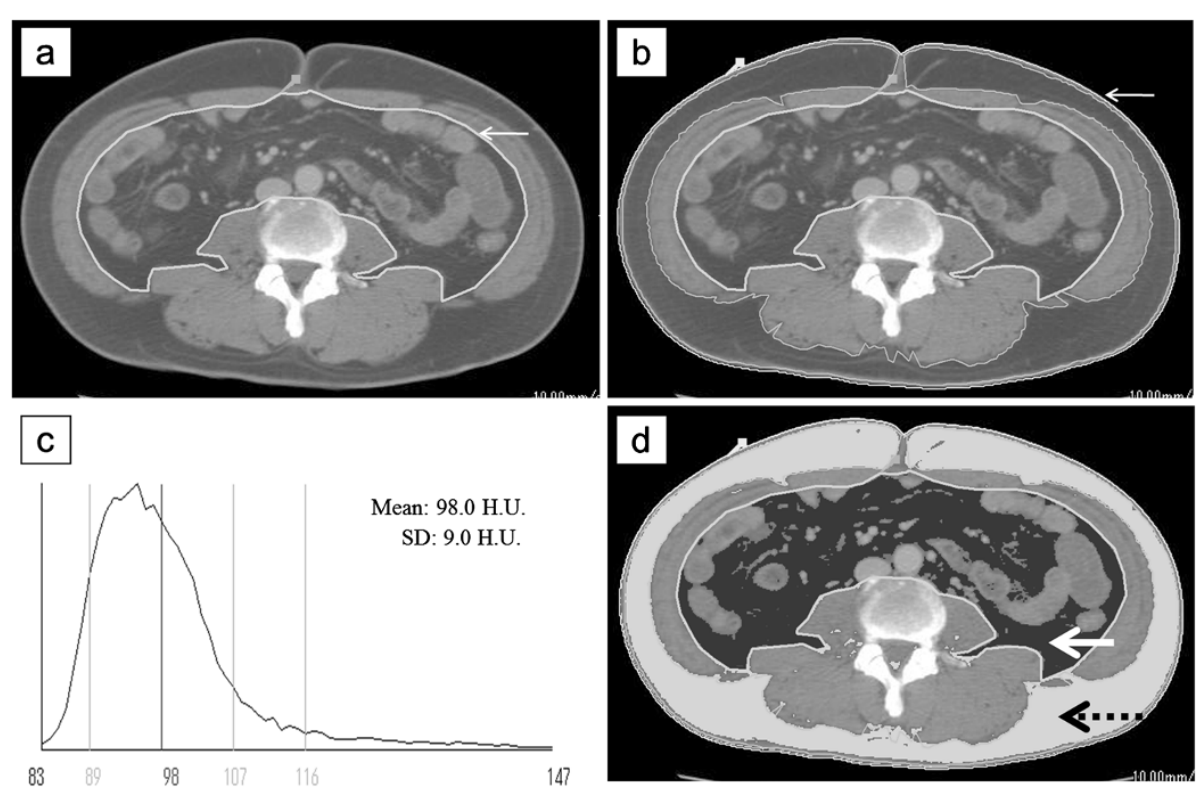

Figure 1 CT images obtained at the navel level demonstrating the method used to determine abdominal fat distribution. (a) The line (arrowed) outlines the intraperitoneal area. (b) The line (arrowed), drawn with a cursor automatically or manually, outlines the subcutaneous fat layer, in which attenuation is measured. (c) A histogram of the CT numbers (in Hounsfield units) in the area outlined in b (mean \pm 2 SD). (d) Measurement of visceral fat tissue (solid arrow). The total fat area is represented by the region outlined by the circumference of the abdominal wall. The visceral fat area was subtracted, and the remainder was regarded as the subcutaneous fat area (dotted arrow). 
Table 1 Comparison of clinical background data of patients who underwent distal gastrectomy-Billroth I and distal gastrectomy-Roux-en-Y

\begin{tabular}{|c|c|c|c|}
\hline & DGBI $n=104$ & DGRY $n=48$ & $P$ \\
\hline Age (years) & 64 (38 to 85$)$ & 62.5 (37 to 80$)$ & $0.2574^{c}$ \\
\hline $\operatorname{Sex}(M / F)$ & $69 / 35$ & $37 / 11$ & $0.1804^{a}$ \\
\hline BMI $\left(\mathrm{kg} / \mathrm{m}^{2}\right)$ before surgery & $22.8(16.0$ to 30.5$)$ & $22.4(17.1$ to 31.0$)$ & $0.7243^{c}$ \\
\hline VFA before surgery $\left(\mathrm{cm}^{2}\right)$ & 75.2 (15.9 to 265.4$)$ & 80.6 (14.6 to 236.7$)$ & $0.9148^{c}$ \\
\hline SFA before surgery $\left(\mathrm{cm}^{2}\right)$ & 122.5 (35.5 to 267.3$)$ & 113.8 (14.9 to 231.5$)$ & $0.4142^{c}$ \\
\hline Albumin level before surgery (mg/dl) & 4.3 (2.5 to 4.8$)$ & 4.1 (2.8 to 4.7$)$ & $0.0128^{c}$ \\
\hline Approach (laparoscopy/laparotomy) & $15 / 89$ & $0 / 48$ & $0.0030^{b}$ \\
\hline Lymph node dissection $(<\mathrm{D} 2 / \geq \mathrm{D} 2)$ & $59 / 45$ & $20 / 28$ & $0.0840^{\mathrm{a}}$ \\
\hline Omentectomy (yes/no) & $13 / 91$ & $15 / 33$ & $0.0056^{\mathrm{a}}$ \\
\hline Pathological T (1/2/3/4) & $74 / 28 / 2 / 0$ & $32 / 12 / 4 / 0$ & $0.4420^{d}$ \\
\hline Pathological N (0/1/2/3) & $78 / 20 / 6 / 0$ & $34 / 10 / 3 / 1$ & $0.5484^{d}$ \\
\hline Pathological stage $(1 / 2 / 3 / 4)$ & $86 / 11 / 7 / 0$ & $37 / 5 / 5 / 1$ & $0.3587^{d}$ \\
\hline Adjuvant chemotherapy (yes/no) & $10 / 94$ & $5 / 43$ & $1.0000^{b}$ \\
\hline Recurrence (yes/no) & $4 / 100$ & $3 / 45$ & $0.5110^{b}$ \\
\hline
\end{tabular}

${ }^{a} X^{2}$ test; ${ }^{b}$ Fisher's exact test; ${ }^{C}$ Wilcoxon rank sum test; ${ }^{d}$ Mann-Whitney $U$ test. $B M I$ body mass index, $D G B I$ distal gastrectomy with Billroth $I, D G R Y$ distal gastrectomy with Roux-en-Y, SFA subcutaneous fat area, VFA visceral fat area.

the CT numbers (in Hounsfield units) for the fat tissue was calculated. A histogram for the fat tissue was computed, based on the mean attenuation \pm 2 SD (Figure 1c). Within the region outlined in Figure 1a, the tissue with attenuation within the mean $\pm 2 \mathrm{SD}$ was considered to be the VFA (white arrow, Figure 1d). The total fat area was calculated in the region outlining the circumference of the abdominal wall. The VFA was subtracted and the remainder was regarded as the subcutaneous fat area (black arrow) (Figure 1d).

\section{Statistical analysis}

Differences between groups were examined for statistical significance using the Student's $t$ test with Yates' correction, the $\chi^{2}$ test, Fisher's exact probability test, Wilcoxon rank sum test, or the Mann-Whitney $U$ test. Univariate analysis was performed to identify the factors that determined visceral fat loss. The identified variables were subsequently entered into a multivariate analysis using logistic regression analysis to identify independent factors that influenced the visceral fat loss. Significance was assumed for $P<0.05$. Statistical analysis was performed using JMP version 9.0 (SAS Institute Inc, Cary, NC).

\section{Results}

\section{Comparison of patients' characteristics according to} surgical procedure

Table 1 compares the background characteristics of patients who underwent DGBI and DGRY. Age, sex, body mass index (BMI), and VFA before surgery were not different among the groups. At baseline, the serum albumin concentration was significantly higher in the DGBI (4.25 \pm $0.36 \mathrm{mg} / \mathrm{dl})$ than in the DGRY group $(4.11 \pm 0.36 \mathrm{mg} / \mathrm{dl}$, $P=0.0317)$

With regard to the surgical factors, there was a significant difference in surgical approach (that is, the proportion of

Table 2 Comparison of indexes of adiposity among patients with distal gastrectomy-Billroth I and distal gastrectomy-Roux-en-Y

\begin{tabular}{|c|c|c|c|}
\hline & DGBI $n=104$ & DGRY $n=48$ & $P$ \\
\hline BMI after surgery & $20.4(15.0$ to 28.1$)$ & 19.7 (16.9 to 26.2$)$ & $0.3789^{a}$ \\
\hline VFA after surgery $\left(\mathrm{cm}^{2}\right)$ & $50.8(6.4$ to 167.1$)$ & 37.4 (11.7 to 144.5$)$ & $0.0742^{\mathrm{a}}$ \\
\hline SFA after surgery $\left(\mathrm{cm}^{2}\right)$ & 81.14 (19 to 230.8 ) & 69.9 (23.5 to 184$)$ & $0.0808^{a}$ \\
\hline Alb after surgery $(\mathrm{mg} / \mathrm{dl})$ & 4.3 (2.4 to 4.7$)$ & 4.2 (3.7 to 4.6$)$ & $0.3065^{\mathrm{a}}$ \\
\hline Reduction rate of BMI (\%) & $8.7(-15$ to 31.2$)$ & $10.8(-2.7$ to 22.5$)$ & $0.1678^{\mathrm{a}}$ \\
\hline Reduction rate of VFA (\%) & $30.6(-130.2$ to 90.1$)$ & $44.2(-14.5$ to 85.2$)$ & $0.0027^{\mathrm{a}}$ \\
\hline Reduction rate of SFA (\%) & $33.0(-124.6$ to 82.3$)$ & $31.8(-147.7$ to 87.4$)$ & $0.5722^{\mathrm{a}}$ \\
\hline
\end{tabular}

Wilcoxon rank sum test. BMI body mass index, DGBI distal gastrectomy with Billroth I, DGRY distal gastrectomy with Roux-en-Y, SFA subcutaneous fat area, VFA visceral fat area. 
Table 3 Results of univariate analysis for visceral fat loss after gastrectomy

\begin{tabular}{lccc}
\hline Univariate analysis & High VFA loss group & Low VFA loss group & \multicolumn{1}{c}{$\boldsymbol{P}$} \\
\hline Sex (M/F) & $53 / 23$ & $53 / 23$ & $1.0000^{\mathrm{a}}$ \\
Adjuvant chemotherapy (yes/no) & $14 / 62$ & $59 / 17$ & $0.0006^{\mathrm{b}}$ \\
Reconstruction (BI/RY) & $45 / 31$ & $49 / 27$ & $0.0146^{\mathrm{a}}$ \\
Lymph node dissection (<D2/ZD2) & $30 / 46$ & $75 / 1$ & $0.0020^{\mathrm{a}}$ \\
Pathological stage (1 to 2/ 3 to 4) & $64 / 12$ & $5 / 71$ & $0.0023^{\mathrm{b}}$ \\
Omentectomy (yes/no) & $23 / 53$ & $11 / 65$ & $0.0003^{\mathrm{b}}$ \\
Surgical approach (laparoscopy/laparotomy) & $4 / 72$ & & $0.1002^{\mathrm{b}}$ \\
\hline
\end{tabular}

${ }^{\mathrm{a}} X^{2}$ test; ${ }^{\mathrm{b}}$ Fisher's exact test; $B I$ Billroth I, $R Y$ Roux-en-Y, VFA, visceral fat area.

patients who underwent laparoscopy versus laparotomy) between the DGBI and DGRY groups $(P=0.0030)$, and in omentectomy between the DGBI and DGRY groups $(P=0.0056)$.

Analysis of the pathological factors and postoperative clinical course showed that there was no significant difference between the groups in pathological T, pathological N, pathological stage, adjuvant chemotherapy, or recurrence.

\section{Comparison of BMI and VFA according to surgical procedure}

Table 2 lists comparative data related to BMI and VFA: BMI after surgery and the rate of reduction of BMI $(\triangle \mathrm{BMI} \%)$ were not different between the DGBI and DGRY groups.

The VFA after DGBI $\left(62.4 \pm 39.8 \mathrm{~cm}^{2}\right)$ was lower than that after DGRY $\left(50.7 \pm 36.2 \mathrm{~cm}^{2}\right)$, but the difference was not statistically significant $(P=0.0742)$.

The rate of reduction of the VFA $(\triangle \mathrm{VFA} \%)$ in the DGBI group $(24.7 \pm 36.8 \%)$ was significantly lower than in the DGRY group $(42.2 \pm 24.1 \%, P=0.0027)$. We also examined $\triangle V F A \%$ in pathological stage I patients. The $\triangle \mathrm{VFA} \%$ in the DGBI group $(21.7 \pm 38.1 \%)$ was significantly lower than in the DGRY group $(39.0 \pm 24.4 \%, P=0.0134)$.

\section{Determinants of postoperative visceral fat loss}

We examined the factors involved in visceral fat loss. For this purpose, the study population was divided into a 'high VFA loss group' and a 'low VFA loss group' using the value of the median $\triangle$ VFA $\%$, which was $36.1 \%$. As shown in Table 3, among the clinicopathological factors examined, adjuvant chemotherapy (yes vs. no, $P=0.0006$ ), type of reconstruction (BI vs. RY, $P=0146$ ), field of lymph node dissection ( $\angle \mathrm{D} 2$ vs. $\geq \mathrm{D} 2, P=0.0020)$, omentectomy (yes vs. no, $P=0.0003)$, and pathological stage $(1 / 2$ vs. $3 / 4 ; P=0.0023)$ correlated significantly with postoperative visceral fat loss. Multivariate logistic regression analysis that included these factors identified reconstruction (BI vs. RY; $P=0.0232$ ) and adjuvant chemotherapy (yes vs. no, $P=0.0330)$ as the significant determinants of visceral fat loss (Table 4).

\section{Discussion}

It is reported that body weight loss is mainly caused by loss of body fat [12]. However, the effects of gastrectomy procedures on postoperative visceral fat have not been thoroughly examined. In this study, we analyzed the effect of surgical procedure on VFA. In the early postoperative period, fat volumes are seriously affected by the metabolic abnormality caused by surgery. Previous studies indicated that body weight loss occurs mainly during the first 3 months after surgery [11]. Therefore, in this study, we compared CT images taken at least 6 months after surgery, when the nutritional status is presumed to be stable [16].

A few studies examined changes in total fat or visceral fat after gastrectomy. Liedman et al. [12] studied changes in body composition by measuring total body potassium and water in 75 patients with gastric cancer. They observed a significant decrease in total body fat at 6 months after surgery but a subsequent marginal recovery at 12 months. In another study, Kiyama et al. [13] evaluated fat mass by multifrequency bioelectrical impedance

Table 4 Results of multivariate analysis for visceral fat loss after gastrectomy

\begin{tabular}{|c|c|c|c|}
\hline Multivariate analysis & Hazard ratio & $95 \%$ confidence interval & $P$ \\
\hline Adjuvant chemotherapy (yes/no) & 5.0290 & 1.1347 to 28.3460 & 0.0330 \\
\hline Reconstruction (BI/RY) & 0.4249 & 0.1965 to 0.8909 & 0.0232 \\
\hline Lymph node dissection (<D2/ $\geq D 2)$ & 0.9539 & 0.4482 to 2.0343 & 0.9022 \\
\hline Pathological stage (1 to $2 / 3$ to 4 ) & 0.4142 & 0.0723 to 2.0248 & 0.2774 \\
\hline Omentectomy (yes/no) & 0.4862 & 0.1364 to 1.5993 & 0.2387 \\
\hline
\end{tabular}

BI Billroth I, RY Roux-en-Y. 
analysis before and after gastrectomy. They found a larger decrease in body fat mass after TG than after subtotal gastrectomy and laparoscopy-assisted gastrectomy. Yoon et al. [17] reported a greater loss of visceral fat tissue after TG than after subtotal gastrectomy at 6 months after surgery. At 12 months after surgery, the losses in BMI, total adipose tissue, subcutaneous, and visceral fat tissues were all greater after TG than after subtotal gastrectomy. Miyato et al. [16] reported that postoperative visceral fat of the RY group tended to be smaller than that of the BI group, although the difference was not statistically significant, perhaps because of the small sample size. Based on these differences, there is a possibility that the reconstruction procedure might have some effects on VFA.

The reasons for the differences in visceral fat reduction between BI and RY could not be elucidated in this retrospective study. Previous studies on bariatric surgery are of some help. It is reported that visceral fat reduction was greater after RY gastric bypass than after vertical banded gastroplasty [18]. Although the mechanisms of fat reduction or improvement in insulin resistance are not completely understood in bariatric surgery, the importance of gut hormones has been reported. Among the various gut hormones, gastric inhibitory polypeptide (GIP) and glucagon-like peptide-1 (GLP-1) are reported to regulate fat metabolism. GIP is released from the duodenal endocrine $\mathrm{K}$ cells immediately after the absorption of fat or glucose [19]. Furthermore, fat intake induces hypersecretion of GIP, which increases nutrient uptake and triglyceride accumulation in adipocytes [20]. In the context of this study, Korner et al. [21] reported lower GIP levels after RY gastric bypass than after adjustable gastric banding, and concluded that the blunted GIP secretion after RY seems to contribute to the greater weight loss and improved glucose homeostasis compared with adjustable gastric banding. GLP-1 is a naturally occurring incretin hormone with a potent blood-glucose lowering action only during hyperglycemia; a GLP-1 analogue reduces visceral fat [22]. Peterli et al. [23] reported higher GLP-1 levels after RY gastric bypass than after sleeve gastrectomy. The GIP level might also be lower and the GLP-1 level might be higher after RY than BI, and fat accumulation is lower after RY than BI. Fat malabsorption might be another reason; since clinical tests after RY showed significantly lower fat absorption than after BI and double-tract reconstruction, which accommodated for the passage of food through the duodenum [24].

As for omentectomy, our data showed that it was not a significant determinant of visceral fat loss by multivariate analysis. There are some reports of surgical removal of visceral fat thorough omentectomy $[25,26]$. There was no significant difference between RY gastric bypass without omentectomy and with omentectomy in fat mass 12 months after surgery [25]; this is compatible with our result.

These results might be helpful in selecting the reconstruction method used after distal gastrectomy, especially for obese patients when remnant stomachs are large enough so that either BI or RY could be performed. However, our study has several limitations. First, a retrospective sample was used in this analysis, and thus the clinical background, such as extent of lymph node dissection, omentectomy, and surgical approach, is different between compared groups, although these factors did not correlate with visceral fat loss in this study. Next, the size of remnant stomach, which could affect the amount of food intake after surgery, might be smaller after RY than after $\mathrm{BI}$, because the former construction technique was often selected when the remnant stomach was too small to allow for anastomosis with the duodenum. Third, the long-term results are unknown, because we examined CT data only 1 year after surgery.

Further prospective studies with stratified randomization and long-term follow-up data, such as VFA after 3 years or 5 years and cause of death, are needed to confirm the effects of reconstruction after gastrectomy on visceral fat.

\section{Conclusions}

Visceral fat loss after RY was larger than that after BI. Further prospective studies are needed to confirm the effects of reconstruction after distal gastrectomy on visceral fat.

\section{Abbreviations}

Bl: Billroth I; BMl: Body mass index; CT: Computed tomography; DGBI: Distal gastrectomy with Billroth I; DGRY: Distal gastrectomy with RouX-en-Y; GIP: Gastric inhibitory polypeptide; GLP-1: Glucagon-like peptide-1; RY: Roux-en-Y; SD: Standard deviation; VFA: Visceral fat area.

\section{Competing interests}

The authors declare that they have no competing interests.

\section{Authors' contributions}

$\mathrm{KT}$ collected data, performed analysis and drafted the manuscript. IM conceived this study, helped to draft the manuscript and participated in the treatment of these patients. MY participated in study design, literature search, and coordination. Ol participated in study design and helped to draft the manuscript. KK, MM, TS, SN, MO, and $\mathrm{HO}$ participated in the treatment of these patients. All authors read and approve the final manuscript.

Received: 11 January 2013 Accepted: 12 May 2013

Published: 21 June 2013

\section{References}

1. Isobe Y, Nashimoto A, Akazawa K, Oda I, Hayashi K, Miyashiro I, Katai H, Tsujitani S, Kodera Y, Seto Y, Kaminishi M: Gastric cancer treatment in Japan: 2008 annual report of the JGCA nationwide registry. Gastric Cancer 2011, 14:301-316.

2. Kunisaki C, Akiyama H, Nomura M, Matsuda G, Otsuka Y, Ono H, Nagahori $Y$, Hosoi H, Takahashi M, Kito F, Shimada H: Significance of long-term follow-up of early gastric cancer. Ann Surg Oncol 2006, 13:363-369.

3. Fujioka S, Matsuzawa Y, Tokunaga K, Tarui S: Contribution of intraabdominal fat accumulation to the impairment of glucose and lipid metabolism in human obesity. Metabolism 1987, 36:54-59. 
4. Fujimoto WY, Newell-Morris LL, Grote M, Bergstrom RW, Shuman WP: Visceral fat obesity and morbidity: NIDDM and atherogenic risk in Japanese American men and women. Int J Obes 1991, 15(Suppl 2):41-44.

5. Marcus MA, Murphy L, Pi-Sunyer FX, Albu JB: Insulin sensitivity and serum triglyceride level in obese white and black women: relationship to visceral and truncal subcutaneous fat. Metabolism 1999, 48:194-199.

6. Lee SE, Lee JH, Ryu KW, Nam B, Kim CG, Park SR, Kook MC, Kim YW: Changing pattern of postoperative body weight and its association with recurrence and survival after curative resection for gastric cancer. Hepatogastroenterology 2012, 59:430-435.

7. Moriwaki Y, Kunisaki C, Kobayashi S, Harada H, Imai S, Kasaoka C: Does body mass index (BMI) influence morbidity and long-term survival in gastric cancer patients after gastrectomy? Hepatogastroenterology 2003, 50:284-288.

8. Armbrecht U, Lundell L, Lindstedt G, Stockbruegger RW: Causes of malabsorption after total gastrectomy with Roux-en-Y reconstruction. Acta Chir Scand 1988, 154:37-41.

9. Bradley EL 3rd, Isaacs J, Hersh T, Davidson ED, Millikan W: Nutritional consequences of total gastrectomy. Ann Surg 1975, 182:415-429.

10. Friess H, Bohm J, Muller MW, Glasbrenner B, Riepl RL, Malfertheiner $P$, Buchler MW: Maldigestion after total gastrectomy is associated with pancreatic insufficiency. Am J Gastroenterol 1996, 91:341-347.

11. Adams JF: The clinical and metabolic consequences of total gastrectomy. I. Morbidity, weight, and nutrition. Scand J Gastroenterol 1967, 2:137-149.

12. Liedman B, Andersson H, Bosaeus I, Hugosson I, Lundell L: Changes in body composition after gastrectomy: results of a controlled, prospective clinical trial. World J Surg 1997, 21:416-420. Discussion 420-421.

13. Kiyama T, Mizutani T, Okuda T, Fujita I, Tokunaga A, Tajiri T, Barbul A: Postoperative changes in body composition after gastrectomy. J Gastrointest Surg 2005, 9:313-319.

14. Japanese Gastric Cancer A: Japanese Classification of Gastric Carcinoma 2nd English edition. Gastric Cancer 1998, 1:10-24.

15. Yoshizumi T, Nakamura T, Yamane M, Islam AH, Menju M, Yamasaki K, Arai T, Kotani K, Funahashi T, Yamashita S, Matsuzawa Y: Abdominal fat: standardized technique for measurement at CT. Radiology 1999, 211:283-286.

16. Miyato H, Kitayama J, Hidemura A, Ishigami H, Kaisaki S, Nagawa H: Vagus nerve preservation selectively restores visceral fat volume in patients with early gastric cancer who underwent gastrectomy. J Surg Res 2012, 173:60-67.

17. Yoon DY, Kim HK, Kim JA, Choi CS, Yun EJ, Chang SK, Lee YJ, Park CH: Changes in the abdominal fat distribution after gastrectomy: computed tomography assessment. ANZ J Surg 2007, 77:121-125.

18. Olbers T, Bjorkman S, Lindroos A, Maleckas A, Lonn L, Sjostrom L, Lonroth H: Body composition, dietary intake, and energy expenditure after laparoscopic Roux-en-Y gastric bypass and laparoscopic vertical banded gastroplasty: a randomized clinical trial. Ann Surg 2006, 244:715-722.

19. Falko JM, Crockett SE, Cataland S, Mazzaferri EL: Gastric inhibitory polypeptide (GIP) stimulated by fat ingestion in man. J Clin Endocrinol Metab 1975, 41:260-265.

20. Miyawaki K, Yamada Y, Ban N, Ihara Y, Tsukiyama K, Zhou H, Fujimoto S, Oku A, Tsuda K, Toyokuni S, Hiai H, Mizunoya W, Fushiki T, Holst JJ, Makino M, Tashita A, Kobara Y, Tsubamoto Y, Jinnouchi T, Jomori T, Seino Y: Inhibition of gastric inhibitory polypeptide signaling prevents obesity. Nat Med 2002, 8:738-742.

21. Korner J, Bessler M, Inabnet W, Taveras C, Holst JJ: Exaggerated glucagonlike peptide- 1 and blunted glucose-dependent insulinotropic peptide secretion are associated with Roux-en-Y gastric bypass but not adjustable gastric banding. Surg Obes Relat Dis 2007, 3:597-601.

22. Jendle J, Nauck MA, Matthews DR, Frid A, Hermansen K, During M, Zdravkovic M, Strauss BJ, Garber AJ: Weight loss with liraglutide, a once-daily human glucagon-like peptide-1 analogue for type 2 diabetes treatment as monotherapy or added to metformin, is primarily as a result of a reduction in fat tissue. Diabetes Obes Metab 2009, 11:1163-1172.

23. Peterli R, Steinert RE, Woelnerhanssen B, Peters T, Christoffel-Courtin C, Gass $M$, Kern B, von Fluee M, Beglinger C: Metabolic and hormonal changes after laparoscopic Roux-en-Y gastric bypass and sleeve gastrectomy: a randomized, prospective trial. Obes Surg 2012, 22:740-748.

24. Takase M, Sumiyama $Y$, Nagao J: Quantitative evaluation of reconstruction methods after gastrectomy using a new type of examination: digestion and absorption test with stable isotope ${ }^{13} \mathrm{C}$-labeled lipid compound. Gastric Cancer 2003, 6:134-141.
25. Fabbrini E, Tamboli RA, Magkos F, Marks-Shulman PA, Eckhauser AW Richards WO, Klein S, Abumrad NN: Surgical removal of omental fat does not improve insulin sensitivity and cardiovascular risk factors in obese adults. Gastroenterology 2010, 139:448-455.

26. Dillard TH, Purnell JQ, Smith MD, Raum W, Hong D, Laut J, Patterson EJ: Omentectomy added to Roux-en-Y gastric bypass surgery: a randomized, controlled trial. Surg Obes Relat Dis 2013, 9:269-275.

doi:10.1186/1477-7819-11-146

Cite this article as: Tanaka et al:: Visceral fat changes after distal gastrectomy according to type of reconstruction procedure for gastric cancer. World Journal of Surgical Oncology 2013 11:146.

\section{Submit your next manuscript to BioMed Central and take full advantage of:}

- Convenient online submission

- Thorough peer review

- No space constraints or color figure charges

- Immediate publication on acceptance

- Inclusion in PubMed, CAS, Scopus and Google Scholar

- Research which is freely available for redistribution

Submit your manuscript at www.biomedcentral.com/submit
C) BioMed Central 